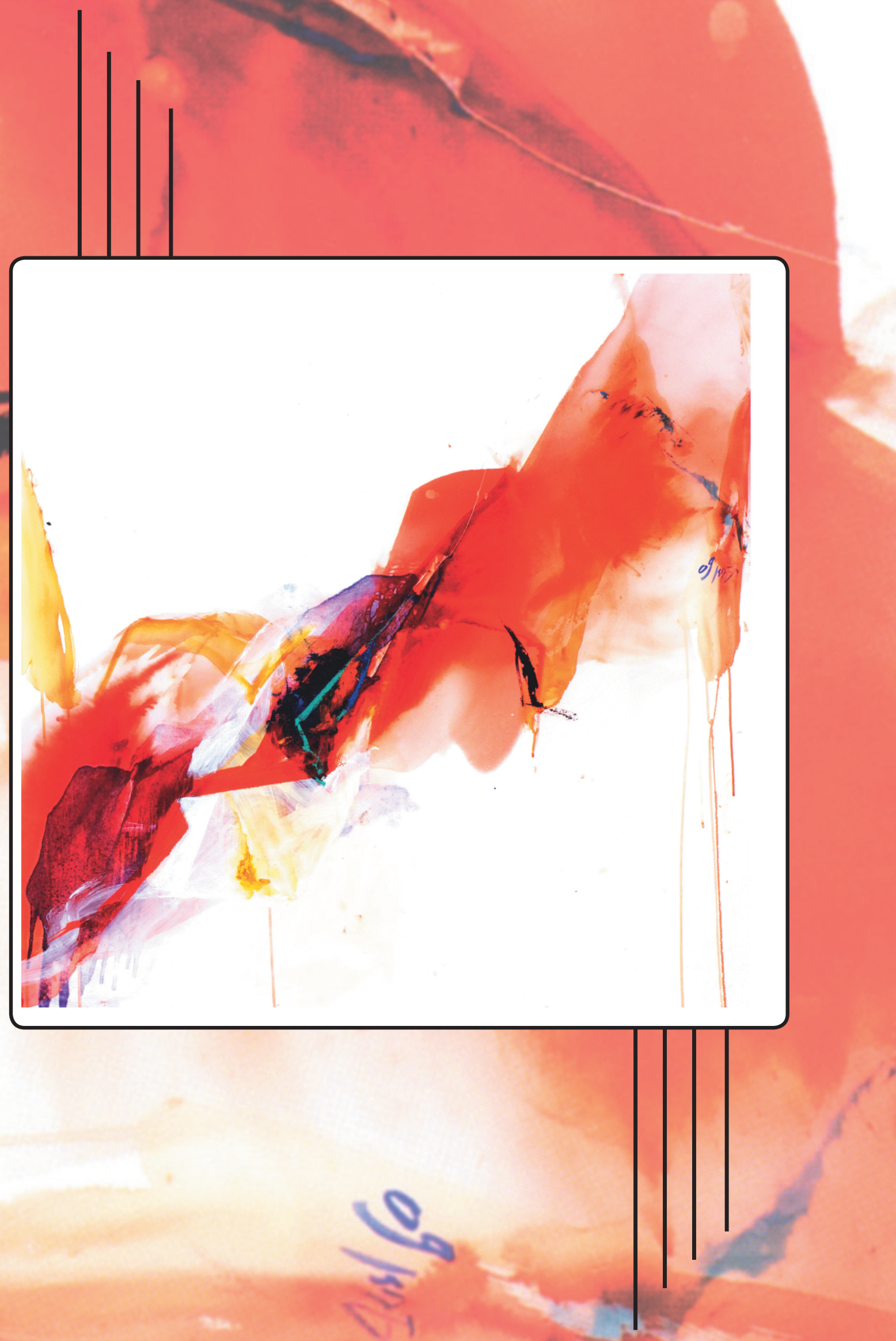


Hommage aux 12 apotres

Óleo sobre Lienzo

$120 \times 120 \mathrm{cms}$

2009

Colección Privada Universidad Santo Tomás 


\title{
DESCENTRALIZACIÓN Y GASTO PÚBLICO SOCIAL EN EDUCACIÓN Y SALUD PARA MUESTRA MUNICIPAL DE SANTANDER 1997 - 2005
}

Amílcar Mojica Pimientol

\begin{abstract}
Resumen
Este documento registra la cobertura de los servicios de educación y salud para una muestra de diecinueve municipios del departamento de Santander en el periodo 1997-2005, analizada bajo el efecto de la política de descentralización implementada en Colombia desde comienzos de los años ochenta. Los resultados más importantes muestran, que los niveles de cobertura para estos dos servicios crecieron de manera importante, la población vulnerable es la mayor beneficiada; en términos de calidad, un manejo más eficiente de los recursos por parte de los gobiernos municipales, permitiría mejorar la calidad en la prestación de estos dos servicios. Así mismo, para el período analizado y como resultado de la implementación de Ley 715 de 2001, se observó un avance efectivo en los niveles de cobertura de la salud y de la educación básica, no obstante para esta última, las tasas de deserción escolar registradas.
\end{abstract}

\section{Palabras Claves}

Descentralización, gasto público, educación, salud.

\begin{abstract}
This document records the coverage of education and health services for a sample of nineteen municipalities of Santander in the period 1997-2005, analyzed under the effect of the decentralization policy implemented in Colombia since the early eighties. The most important results show that coverage levels for these two services grew significantly, with vulnerable populations to gain maximum benefit, in terms of quality, more efficient management of resources by local governments, would improve the quality in providing these two services. Likewise, for the period under review and as a result of the implementation of Act 715 of 2001, there was a great advance in levels of health coverage and basic education, however for the latter, the dropout rates registered.
\end{abstract}

\section{Key words}

Decentralization policy, public asset, education, health.

1 Maestría en Ciencias Económicas. Docente Universidad Santo Tomás Bucaramanga. 


\section{Introducción}

Para las regiones es de vital importancia saber cómo se invierten los recursos públicos en la prestación de los servicios de educación y salud, y si estos procuran el bienestar de la población más vulnerable en términos de calidad y cobertura; en el desarrollo de la presente investigación se plantea el siguiente cuestionamiento, ¿Ha estimulado el proceso de descentralización implementado en nuestro país, la acumulación de recursos a nivel municipal vía transferencias, de tal manera que estos hayan asegurado a través del gasto público social, una mayor cobertura en la prestación del servicio de educación y salud?.

El proceso metodológico utilizado para la escogencia de los 19 municipios de la muestra, tuvo en cuenta una variable muy importante, la concentración de los recursos a nivel del presupuesto municipal, donde los ingresos sumaron durante el periodo de análisis cerca del 71\% del total de ingresos de los 87 municipios del departamento de Santander y por el lado de los gastos el 69\% del global departamental, esta elección nos permitió delimitar el tema en términos de extensión y su posterior análisis. La información referente a las transferencias corrientes de la nación fue tomada del DNP; la del gasto público, fue suministrada por el Banco de la República, entidad que clasifica las cuentas de ingreso y gasto de las Entidades Territoriales de sus respectivas ejecuciones presupuestales; los resultados para los dos sectores estudiados, se obtuvieron tras una dedicada labor de campo, que incluyó una revisión detallada de los documentos producidos por las secretarías de educación y salud del departamento de Santander; esta información sirvió para el cálculo de algunos indicadores de actividad al interior de los sectores, los cuales complementaron el análisis final sobre la gestión desarrollada por los entes municipales.

\section{La descentralización y su evolución}

El proceso de descentralización en Colombia toma como base los principios sobre la cual descansa la teoría de la eficiencia local y de la movilidad de los individuos entre regiones propuesta por Tiebout en el año de 1956; este afirmaba que en ausencia de externalidades, la provisión de bienes públicos a nivel local trae consigo grandes beneficios en términos de eficiencia y bienestar; así, la descentralización permite mejorar la eficiencia en la asignación de recursos, al promover una mejor coordinación entre las decisiones de gasto público y las preferencias de los ciudadanos; esto significa, que las personas revelan sus preferencias al poder emigrar de una región a otra, saliendo de aquellas que no ofrecen oportunidades y desplazándose a las que sí las ofrecen; por lo tanto, es de esperar que la provisión de servicios públicos por parte de las autoridades locales mejore tanto la eficiencia técnica como la de asignación.

Así, la competencia entre las regiones y la libre elección del consumidor le permite establecer la mezcla ideal entre bienes y servicios públicos e impuestos; la región 
que realice la mejor combinación conseguirá para si los factores más productivos, alcanzando un nivel adecuado de oferta en lo referente a bienes y servicios privados y públicos para su comunidad en sus respectivos mercados.

Al respecto comentaba el BID, que la "política de descentralización" debería diseñarse teniendo en cuenta las fuerzas que intervienen en el proceso de cambio estructural que conducen al desarrollo económico"; es decir, que se deben remover las estructuras que no permiten que los mercados funcionen adecuadamente"; en otras palabras, emprender la Reforma del Estado.

Las entidades descentralizadas prácticamente se nutren con las transferencias del gobierno central y son las encargadas de proveer los servicios públicos que proporciona el estado. Entonces, resulta interesante evaluar si los cambios institucionales orientados a dar mayor autonomía a los departamentos y municipios han tenido algún impacto en el suministro de bienes públicos en materia de calidad y cobertura; de esta manera las transferencias del gobierno central hacia los entes territoriales se constituyen en el mecanismo transmisor de la descentralización.

Con la descentralización implementada en Colombia en la década del noventa se querían lograr dos objetivos; fortalecer la legitimidad del estado y aumentar la eficiencia en la asignación de recursos; no obstante afirmaba IREGUI (2001) refiriéndose a la constitución y sus leyes reglamentarias: "parece que el principal objetivo fue mejorar la eficiencia del gasto público nacional delegando su ejecución en las regiones".

La descentralización tiene ventajas en cuanto a mayor eficiencia y legitimidad, pero también es cierto que presenta sus desventajas, entre ellas la desbalanceada distribución de los recursos entre las regiones, lo cual podría crear una creciente disparidad económica entre las entidades descentralizadas; Prudhomme (1995) afirmaba al respecto, que un sector público centralizado intentará realizar una distribución más balanceada, canalizando los recursos de las regiones ricas hacia las pobres. Sin embargo, los sistemas centralizados de gobierno podrían crear distribuciones desiguales favoreciendo regiones políticamente más importantes.

En el nuevo contexto de descentralización implementado en nuestro país, el tema de mercado elimina los subsidios a la oferta e incluye el concepto de subsidios a la demanda; es decir, cambia el concepto de financiar a los pobres, en detrimento de la financiación de las instituciones prestadoras de los servicios públicos. Con el concepto de subsidio a la demanda, se está obligando al autofinanciamiento de las instituciones y esto tiene mucho que ver con el concepto de la focalización; con esta nueva herramienta, se logra identificar a quien se debe dirigir el subsidio (al pobre), de tal manera que se logran satisfacer las necesidades no satisfechas; el focalizar el gasto ayuda a los gobiernos a mejorar los problemas de manejo presupuestal, evitándoles la tarea de reasignación de recursos. 
La idea central de la focalización es que la concentración de los recursos aumenta la eficiencia de las transferencias destinadas a combatir la pobreza. Por eso, la ventaja de focalizar el gasto como estrategia de atención a la población más vulnerable, permite atacar la pobreza en su base, y para conseguirlo es necesario el diseño de programas bien estructurados que permita que en un mundo donde los recursos son escasos, no se malgasten y que los segmentos de la población más necesitada sean al final los más beneficiados. (Banco Mundial 1990, p.3).

"El fundamento conceptual de la focalización se puede tomar de la teoría de la justicia de Rawls (1971), que plantea el principio de maximizar el bienestar del peor (maximin). Esta idea es tomada por Amartya Sen, al señalar que debe dar tratamiento preferente a los más débiles, para garantizar la igualdad, a lo que agrega igualdad de oportunidades" (Giraldo 2005, p.61).

En suma, dirigir recursos públicos a los más pobres, respetando las reglas del mercado; además, si la pobreza fuera el resultado de falencias de la generación de ingresos, la política social debería buscar soluciones al problema, y esa, no es su razón de ser como tal.

La política social debe estar dirigida a los pobres (excluidos) de manera primordial. La exclusión social como tal, reúne a todos aquellos grupos de la sociedad que son rechazados, y sencillamente se les niega la posibilidad de disfrutar de los beneficios institucionales, sociales y culturales alcanzados por una comunidad. Como la política social se basa en la atención de los excluidos, es preciso diseñar estrategias que permitan su inserción al mercado; esto necesariamente "obliga" al pobre a ser agente activo en la búsqueda de soluciones de sus problemas; solo así, se pueden crear condiciones para salir de la pobreza en la cual se encuentra inmerso.

En el año de 1983 el proceso de descentralización da sus primeros avances con el fortalecimiento de los municipios, para lo cual fue necesario examinar la redefinición de las relaciones entre la administración local y el gobierno nacional, estas primeras revisiones generaron al interior del estado una posición de mayor autonomía a los entes territoriales, al trasladarles funciones y recursos desde el nivel central; en términos políticos, se determinó la elección popular de alcaldes; en el fiscal, se fortalecieron los recursos propios de los entes locales y se transfirió el IVA a los municipios, estas acciones estuvieron amparadas por las leyes 14 de 1983 y 12 de 1986.

Es importante señalar que a comienzos de los años noventa y con la expedición de la nueva carta magna, los departamentos y entes municipales de nuestro país, experimentaron un significativo avance en materia de recursos vía transferencias de la nación, estos cambios fueron de hecho beneficiosos para los municipios en materia de educación y salud, ya que se pudieron dar comienzo a una serie de programas especiales que buscaron aumentar la cobertura en los servicios de educación y salud. 
El primer avance significativo en el proceso de descentralización se logró inicialmente con la ley 60 de 1993, gracias a la cual las transferencias a los departamentos para salud y educación aumentaron notablemente (de 15\% de los ingresos gubernamentales en 1993 a 24.5\% de 1996 en adelante); para los municipios se incrementaron del 15\% de los ingresos gubernamentales en 1994 al 17\% en 1997, y al $22 \%$ en el 2001.

Para el caso específico de la educación, los principios fundamentales para el sistema educativo colombiano fueron consignados en la Carta Magna de 1991. Como fruto de este mandato y en base de un amplio proceso de concertación se procedió a la formulación de la ley 115 de 1994, conocida como la Ley General de la Educación. En desarrollo de esta ley y complementarias a la ley 30 de 1992 que organizó el servicio público de la educación superior y la ley 60 de 1993 de distribución y competencias y recursos entre los diferentes entes territoriales del país, se ha producido la reglamentación y normatividad pertinente según los diferentes tópicos, para los niveles educativos y las poblaciones demandantes del servicio educativo ${ }^{2}$.

Tabla 1. Transferencias Sector Educación 1994-2005

Millones de pesos

\begin{tabular}{rrrrrr}
\hline & \multicolumn{1}{c}{ Colombia } & Variación \% & \multicolumn{1}{c}{ Santander } & Variación \% & Participación \% \\
\hline 1994 & 4.020 .031 & $\ldots$ & 226.446 & $\ldots$ & 5,6 \\
1995 & 4.369 .958 & 8,7 & 245.219 & 8,3 & 5,6 \\
1996 & 4.734 .244 & 8,3 & 269.826 & 10,0 & 5,7 \\
1997 & 4.743 .484 & 0,2 & 259.958 & $-3,7$ & 5,5 \\
1998 & 4.732 .650 & $-0,2$ & 249.756 & $-3,9$ & 5,3 \\
1999 & 5.798 .026 & 22,5 & 290.375 & 16,3 & 5,0 \\
2000 & 5.138 .469 & $-11,4$ & 246.086 & $-15,3$ & 4,8 \\
2001 & 6.075 .303 & 18,2 & 290.387 & 18,0 & 4,8 \\
2002 & 7.951 .846 & 30,9 & 446.207 & 53,7 & 5,6 \\
2003 & 8.138 .478 & 2,3 & 436.638 & $-2,1$ & 5,4 \\
2004 & 8.369 .329 & 2,8 & 422.189 & $-3,3$ & 5,0 \\
2005 & 7.357 .282 & $-12,1$ & 351.354 & $-16,8$ & 4,8 \\
Prom & $\mathbf{5 . 9 5 2 . 4 2 5}$ & $\mathbf{5 , 9}$ & $\mathbf{3 1 1 . 2 0 3}$ & $\mathbf{5 , 1}$ & $\mathbf{5 , 3}$ \\
\hline
\end{tabular}

Fuente: Planeación Nacional. Cálculos Autor

La descentralización en términos legales para el sector de la salud comienza en los años noventa, cuando el ministerio del ramo hizo obligatorio transferir, en un período de cinco años, los establecimientos, el personal y el presupuesto de atención de salud primaria a las municipalidades y la atención de salud secundaria y terciaria

2 Informe Nacional sobre el desarrollo de la educación en Colombia. $46^{\text {a }}$. Conferencia Internacional de Educación (CIE). Ginebra Suiza, septiembre 5 al 7de 2001. Bogotá, D.C. Colombia Junio de 2001. 
a los gobiernos departamentales; sin embargo, esta situación se vio afectada por elementos inherentes al proceso, tales como requisitos onerosos de certificación y recursos insuficientes para pagar el costo de la descentralización; Con el aumento de las transferencia vía ley 60 de 1993, se logra un avance muy significativo, gracias a la cual las mismas a los departamentos para salud aumentaron de manera importante; no obstante, dichas transferencias guardaban poca relación con las necesidades de la salud locales o con el costo de los servicios; meses después aparecería la ley 100 de 1993 cuyo espíritu fue sumamente descentralizado.

A continuación, el Acto Legislativo 01 de 2001 modificó los artículos 356 y 357 de la Constitución nacional, mientras la ley 715 de 2001 determinó la forma de distribución de los recursos; esta ley intentó solucionar los problemas derivados de la ley 60 de 1993, especialmente en los aspectos del aumento excesivo del gasto en las entidades territoriales, la volatilidad de las transferencias y la duplicidad de funciones que se presentaba entre los departamentos y los municipios en la provisión de los bienes públicos locales.

Tabla 2. Transferencias Sector Salud 1994-2005

Millones de pesos

\begin{tabular}{rrrrrr}
\hline & \multicolumn{1}{c}{ Colombia } & Variación \% & Santander & Variación \% & Participación \% \\
\hline 1994 & 1.568 .556 & $\ldots$ & 87.365 & $\ldots$ & 5,6 \\
1995 & 1.952 .607 & 24,5 & 104.566 & 19,7 & 5,4 \\
1996 & 2.157 .355 & 10,5 & 115.560 & 10,5 & 5,4 \\
1997 & 2.341 .612 & 8,5 & 122.963 & 6,4 & 5,3 \\
1998 & 2.464 .988 & 5,3 & 122.871 & $-0,1$ & 5,0 \\
1999 & 2.779 .851 & 12,8 & 134.625 & 9,6 & 4,8 \\
2000 & 2.629 .681 & $-5,4$ & 130.004 & $-3,4$ & 4,9 \\
2001 & 3.007 .281 & 14,4 & 148.967 & 14,6 & 5,0 \\
2002 & 3.330 .260 & 10,7 & 158.851 & 6,6 & 4,8 \\
2003 & 3.408 .422 & 2,3 & 151.095 & $-4,9$ & 4,4 \\
2004 & 3.505 .104 & 2,8 & 153.097 & 1,3 & 4,4 \\
2005 & 3.233 .102 & $-7,8$ & 143.431 & $-6,3$ & 4,4 \\
Prom & $\mathbf{2 . 6 9 8 . 2 3 5}$ & $\mathbf{6 , 6}$ & $\mathbf{1 3 1 . 1 1 6}$ & $\mathbf{4 , 5}$ & $\mathbf{4 , 9}$ \\
\hline
\end{tabular}

Fuente: Planeación Nacional. Cálculos Autor.

Sin duda, los cambios enmarcados por la ley 715 de 2001 fueron determinantes en el comportamiento de los ingresos y gastos del sector público nacional, departamental y municipal para los sectores de educación y salud; los efectos más significativos provocados por esta ley en lo referente a funciones y recursos, se comenzaron a sentir en el departamento de Santander y los municipios de la muestra a partir del año 2002, en el cual los recursos transferidos por la nación comienzan a crecer de manera significativa a nivel nacional y local y los resultados en materia de prestación de los servicios de educación y salud en cuanto a cobertura mejoraron de manera importante. Los cambios en materia de transferencias para el departamento de Santander y su comparativo frente al total nacional, se detallan en los cuadros aquí presentados. 


\section{La descentralización y sus resultados a nivel municipal}

A finales del siglo XIX, surge la necesidad del estado por consolidar e integrar al país, motivo por el cual se da inicio a un proceso de centralización que buscó integrar regiones y mercados en aras de la modernización; no obstante, este primer esfuerzo fue poco a poco consolidando un estado ineficiente, cuyas decisiones políticas fueron excesivamente centralistas; este proceso fue el resultado de la subordinación política, administrativa y fiscal de las entidades territoriales al gobierno central, plasmadas en la constitución de 1886.

El estado colombiano registró durante la mayor parte del siglo XX un proceso continuo de centralización en lo político, administrativo y económico; solo a finales de los años setenta se observan tendencias de cambio. Los resultados poco favorables obtenidos durante más de noventa años de centralización del estado, fueron argumentos suficientes para fortalecer el nuevo proceso descentralista, el cual sería el encargado de abrir la puerta al nuevo modelo de desarrollo económico mundial llamado globalización.

En 1973, cerca del 13\% de los ingresos corrientes del gobierno central se transfirieron a los gobiernos locales. En 2001, el indicador comparable fue casi del $50 \%$. Pocos países en el mundo han pasado de ser esencialmente centralizados a ser descentralizados (en este sentido) en tan corto tiempo o tan drásticamente como lo hizo Colombia en el último tercio del siglo XX. (Acosta-Bird, 2003, p.5)

En la década de los setenta, el sistema de transferencia comenzó a mostrar algunos inconvenientes, pocos recursos llegaban a las regiones y los mismos no eran utilizados de manera eficiente; ante este panorama se crea la Misión de Finanzas Intergubernamentales, la cual en su informe final se encarga de afirmar que el sistema existente de transferencia no contemplaba las necesidades fiscales o la capacidad o el esfuerzo fiscal, proponiendo una nueva fórmula para el cálculo del situado fiscal, el cual incluía elementos más adecuados.

En sus comienzos, la descentralización fue considerada como un logro político que generó una gran expectativa en diversos sectores de la población; esta estrategia, fue uno de los cambios políticos más importantes en la segunda mitad del siglo pasado, que respondió a una crisis generalizada del sistema centralizado político colombiano, agobiado por su condición autoritaria, clientelista y cada vez más deshonesta; en ese sentido, fue un proceso eminentemente político que entregó poder a los entes subnacionales para manejar con autonomía relativa sus propios asuntos.

Las transferencias giradas por la nación se han convertido de tiempo atrás en la principal fuente de financiación de los programas de gasto público a nivel municipal, por consiguiente, han asumido el papel de ser el mecanismo transmisor de la descentralización, capaz de cumplir los objetivos trazados en materia de gasto público en los sectores de educación y la salud. 
El Departamento de Santander está situado al noreste del país en la región andina, cuenta con una superficie de $30.537 \mathrm{~km} 2$ lo que representa el $2.7 \%$ del territorio nacional. Cuenta con 87 municipios, distribuidos en 6 provincias a saber: Soto, García Rovira, Guanentá, Mares, Comunera y Vélez y como ciudades cabecera de provincia en su orden, Bucaramanga, Málaga, San Gil, Barrancabermeja, Socorro y Vélez. Bucaramanga oficia como capital del departamento de Santander por norma de la Constitución de 1886.

Por el lado de las transferencias, los resultados muestran que dentro del total girado por la nación a los municipios de la muestra para los sectores de educación y salud, el mayor porcentaje de las mismas se concentró en los municipios de Bucaramanga, Barrancabermeja y Floridablanca; con un acumulado promedio para los tres en el período $1997-2005$, del $92 \%$ y $68.8 \%$ en su orden.

Gráfico 1. Transferencias vs Gastos en Educación

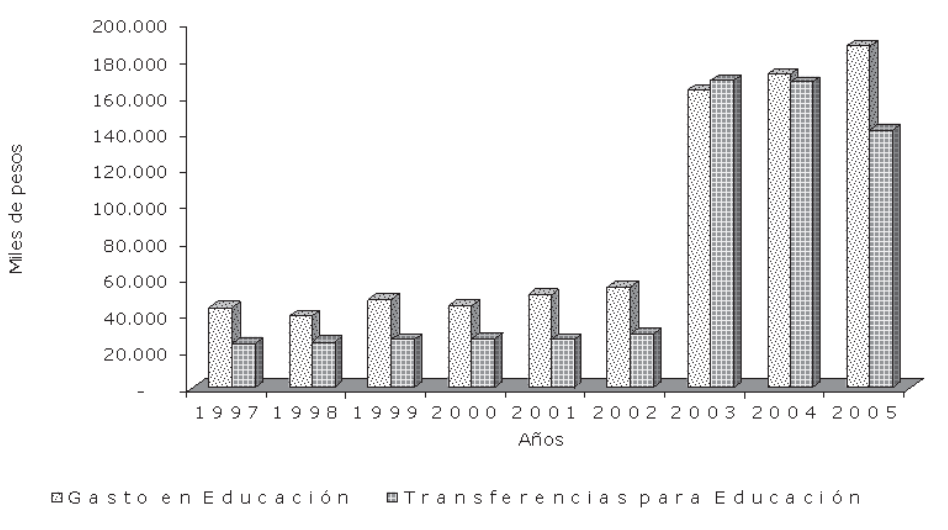

Fuente: Elaboración propia.

Por el lado del gasto público Bucaramanga, Barrancabermeja y Floridablanca ejecutaron en promedio durante el período referenciado el $89.7 \%$ y $73.2 \%$ en educación y salud. La concentración de los ingresos y de los gastos en cabeza de los tres principales municipios del departamento de Santander, refuerza el principio del por qué las regiones políticamente más importantes, son al final las más beneficiadas en materia de recursos. 
Gráfico 2. Tranferencias vs gastos en salud

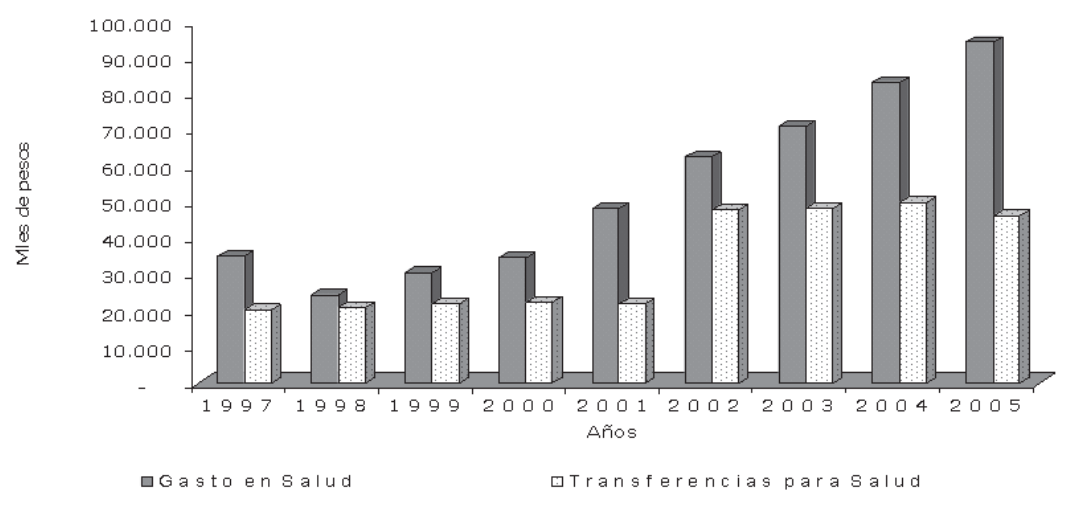

Fuente: Elaboración propia

Los resultados por sectores a nivel global para los diferentes municipios de la muestra, mostraron avances significativos; la cobertura en educación aumentó para los tres niveles básicos de enseñanza durante el período 1997-2005, en preescolar se pasó del $82 \%$ al $88 \%$, la básica primaria se ubicó cerca al $100 \%$ y la secundaria lo hizo entre el $72 \%$ y $86 \%$.

En salud, la cobertura del régimen subsidiado por municipios mostró un comportamiento muy favorable, durante este período se acumularon recursos más que suficientes que fueron invertidos en programas específicos de atención en salud, con lo cual se buscó atender a una franja más amplia de la población vulnerable; los resultados mostraron que Floridablanca entre los tres mayores, fue el de menor crecimiento durante el período, alcanzando al finalizar el mismo, 36\% de cobertura en promedio, mientras que Bucaramanga y Barrancabermeja, alcanzaron el $48.1 \%$ y $59.1 \%$ en su orden.

El aumento de cobertura en la prestación de los servicios en educación y salud a nivel municipal, guarda una relación muy estrecha con el crecimiento de las transferencias giradas por la nación, las cuales adquieren su real importancia con la expedición de la ley 715 de 2001; así, el esfuerzo realizado por el Gobierno Central Nacional en materia de descentralización del gasto público vía transferencias corrientes, condujo a un aumento en los niveles de gasto y por ende en los de cobertura para el caso de los servicios de educación y salud a nivel municipal, alcanzando a su vez una leve mejora en la calidad en la prestación de los mismos. 
Tabla 3. Tasa de cobertura bruta en Educación Básica Departamental

\begin{tabular}{|c|c|c|c|c|c|c|}
\hline DEPARTAMENTOS & 2001 & 2002 & 2003 & 2004 & 2005 & 2006 \\
\hline Sucre & $112,66 \%$ & $113,93 \%$ & $123,38 \%$ & $114,61 \%$ & $115,04 \%$ & $111,77 \%$ \\
\hline Córdoba & $108,54 \%$ & $113,81 \%$ & $107,03 \%$ & $107,25 \%$ & $105,71 \%$ & $109,27 \%$ \\
\hline Casanare & $96,34 \%$ & $99,31 \%$ & $106,19 \%$ & $105,30 \%$ & $104,91 \%$ & $105,16 \%$ \\
\hline Meta & $98,41 \%$ & $99,11 \%$ & $102,52 \%$ & $102,93 \%$ & $105,85 \%$ & $104,41 \%$ \\
\hline Quindío & $98,19 \%$ & $99,86 \%$ & $106,88 \%$ & $101,26 \%$ & $103,34 \%$ & $103,15 \%$ \\
\hline Bolívar & $97,47 \%$ & $107,42 \%$ & $102,44 \%$ & $101,72 \%$ & $104,44 \%$ & $101,34 \%$ \\
\hline Magdalena & $100,20 \%$ & $107,63 \%$ & $108,60 \%$ & $103,76 \%$ & $102,94 \%$ & $101,07 \%$ \\
\hline Antioquia & $96,87 \%$ & $101,38 \%$ & $100,91 \%$ & $97,93 \%$ & $100,05 \%$ & $101,00 \%$ \\
\hline Caldas & $96,27 \%$ & $99,57 \%$ & $100,09 \%$ & $100,67 \%$ & $100,61 \%$ & $100,42 \%$ \\
\hline Caquetá & $98,96 \%$ & $97,62 \%$ & $97,84 \%$ & $99,79 \%$ & $100,19 \%$ & $99,72 \%$ \\
\hline Risaralda & $96,69 \%$ & $99,26 \%$ & $99,74 \%$ & $99,84 \%$ & $99,07 \%$ & $99,65 \%$ \\
\hline Cauca & $93,04 \%$ & $95,97 \%$ & $97,34 \%$ & $99,15 \%$ & $98,04 \%$ & $98,65 \%$ \\
\hline Huila & $99,17 \%$ & $101,66 \%$ & $102,35 \%$ & $100,44 \%$ & $98,77 \%$ & $98,02 \%$ \\
\hline Norte Santander & $99,85 \%$ & $98,48 \%$ & $95,92 \%$ & $96,53 \%$ & $97,48 \%$ & $96,74 \%$ \\
\hline Boyacá & $98,38 \%$ & $100,12 \%$ & $101,00 \%$ & $96,50 \%$ & $97,89 \%$ & $96,73 \%$ \\
\hline Cesar & $100,76 \%$ & $102,79 \%$ & $101,22 \%$ & $97,40 \%$ & $98,60 \%$ & $96,37 \%$ \\
\hline Valle & $99,32 \%$ & $91,06 \%$ & $91,51 \%$ & $96,92 \%$ & $98,76 \%$ & $96,06 \%$ \\
\hline Bogotá DC & $90,99 \%$ & $90,77 \%$ & $93,39 \%$ & $90,49 \%$ & $97,40 \%$ & $95,79 \%$ \\
\hline Putumayo & $109,21 \%$ & $111,29 \%$ & $100,85 \%$ & $91,99 \%$ & $92,92 \%$ & $93,41 \%$ \\
\hline Arauca & $101,44 \%$ & $103,28 \%$ & $94,36 \%$ & $90,12 \%$ & $93,49 \%$ & $91,88 \%$ \\
\hline Santander & $92,24 \%$ & $94,49 \%$ & $96,40 \%$ & $97,19 \%$ & $95,82 \%$ & $91,72 \%$ \\
\hline Nariño & $97,66 \%$ & $99,24 \%$ & $100,42 \%$ & $97,08 \%$ & $90,51 \%$ & $91,02 \%$ \\
\hline Tolima & $100,11 \%$ & $98,02 \%$ & $102,40 \%$ & $98,76 \%$ & $99,81 \%$ & $89,45 \%$ \\
\hline Cundinamarca & $98,41 \%$ & $99,24 \%$ & $99,51 \%$ & $96,07 \%$ & $98,22 \%$ & $89,41 \%$ \\
\hline Atlántico & $92,55 \%$ & $96,93 \%$ & $94,57 \%$ & $89,54 \%$ & $91,51 \%$ & $84,70 \%$ \\
\hline La Guajira & $87,70 \%$ & $91,09 \%$ & $99,43 \%$ & $82,93 \%$ & $76,89 \%$ & $78,83 \%$ \\
\hline San Andrés & $88,43 \%$ & $86,32 \%$ & $82,60 \%$ & $81,95 \%$ & $80,07 \%$ & $78,34 \%$ \\
\hline Chocó & $91,19 \%$ & $91,91 \%$ & $85,85 \%$ & $75,80 \%$ & $75,81 \%$ & $77,86 \%$ \\
\hline
\end{tabular}

Fuente: DANE - EDUCACION FORMAL. Población: DANE - CENSO 2005

En el sector educativo, la deserción escolar es un fenómeno complejo que deriva de múltiples causas que explican la inasistencia a la escuela. Las más comunes se ubican en las restricciones de demanda. Un alto porcentaje de los jóvenes en el decil de ingresos más bajo (los más pobres) no asiste a la escuela. A nivel nacional la tasa 
de deserción se movió entre el 7.6\% y 6.0\% para el período 1997-2005; no obstante, a nivel del departamento de Santander, la tasa cerró al año 2005 en un 5.0\%, un punto menos que el registro nacional.

En algunos municipios del departamento de Santander la deserción escolar ha disminuido y la cobertura ha venido aumentando a raíz de algunas estrategias adoptadas, entre las cuales sobresale el beneficio a los costos educativos, los bibliobancos, el subsidio de transporte, la alimentación escolar y otros aspectos como kits escolares, entrega de dotación y uniformes.

Por esta razón el tema de la deserción comenzó a ceder, ya que uno de los factores de la misma para las familias de escasos recursos, eran los costos educativos; no obstante, subsisten otros elementos adicionales que intervienen en los jóvenes, entre los que se destacan el interés de salir a trabajar y la falta de un acompañamiento y orientación de los padres.

En este orden, las soluciones a la deserción escolar requieren de una estrategia a nivel integral que contemple un sin número de factores tanto al interior como al exterior del sistema educativo; así, se hace necesario el uso más racional y eficiente de los recursos asignados para la educación, de esta forma las instituciones educativas pueden contar con los medios económicos, humanos y materiales suficientes para alcanzar niveles de calidad más elevados.

Para mejorar la pertinencia y la calidad de prestación del servicio educativo a las poblaciones vulnerables y combatir la deserción se han venido implementado modelos educativos y de formación de los docentes, que no son otra cosa que modelos educativos flexibles de inversión en infraestructura escolar, con apoyo del Ministerio de Educación Nacional, para lo cual se han determinado políticas que amplían la cobertura bruta total de transición, básica y media superior; así mismo, otras que buscan fortalecer la formación técnica y tecnológica con base en la normatividad vigente y pretenden disminuir la tasa de deserción estudiantil, creando estrategias como los programas PER (Programa de Educación Rural) y el POS PRIMARIA que amplía la cobertura y disminuye la deserción, este último programa POS PRIMARIA tiene como eje la educación en la población rural, la cual se ha ampliado hasta el noveno grado $\left(9^{\circ}\right)$. Así mismo, con el análisis y uso de resultados de las evaluaciones para los estudiantes, el estado ha diseñado las Pruebas SABER (5․ y $9^{\circ}$. Grados) y las pruebas de Estado (11 ${ }^{\circ}$. Grado) que buscan mejorar la calidad de la oferta y la pertinencia de los programas de educación preescolar, básica y media.

Otras de las políticas diseñadas en busca de la excelencia educativa está relacionada con la creación del programa Banco de objetos virtuales de educación superior y la televisión con fortalecimiento en la formación técnico y tecnológico, la cual busca aumentar la oferta educativa de los Centros Regionales de Educación Superior. 
En lo referente a la estrategia de la formación docente, esta se ha venido fortaleciendo en competencias básicas y en el uso de medios para el mejoramiento de la formación inicial de los docentes a través de la acreditación de la alta calidad de las Escuelas Normales Superiores; así mismo, se está contemplando la idea de mejorar la calidad de los docentes en una segunda lengua, específicamente en el aprendizaje del idioma inglés.

Como complemento a estos programas, se debe desarrollar una política integral de atención a la niñez y la juventud que contemple no sólo el aspecto educativo sino también aspectos relacionados con la salud, la estructura alimenticia de los niños y el desarrollo de habilidades especiales. Un proyecto así contemplado, puede obtener en el corto y mediano plazo los resultados deseados. Por ello, no se debe dejar de lado los factores del entorno que pueden afectar la condición de la familia y la comunidad; se requiere que todos los actores sociales, entre ellos los mismos jóvenes, asuman su papel como parte del engranaje social llamado a construir el desarrollo del país.

En síntesis, el estado debe realizar inspección en los procesos educativos que favorezcan la calidad y mejoren la educación, estableciendo responsabilidades para determinar criterios y procedimientos; de igual manera, debe propender por un buen ambiente escolar que unido a condiciones mínimas de bienestar económico y social en la familia sean los elementos indispensables para el éxito de los escolares, y la responsabilidad de que esto ocurra, tal como lo expresa la Constitución recae en la familia, la sociedad y el Estado.

El Sistema de Salud desde los inicios de los años 70, era un sistema financiado directamente por el estado colombiano y sus acciones estaban enfocadas únicamente a la curación, tratamiento y rehabilitación del paciente. En términos de la calidad en la prestación del servicio de salud, hasta hace algunos años, la misma se asociaba a procesos de atención a la enfermedad. Así, todas las acciones del sector se relacionaban con atender enfermos, concentrando los esfuerzos en la construcción de hospitales, contratación de personal y adquisición de tecnologías para tratar médicamente patologías o enfermedades que pusieran en riesgo la vida de las personas.

Es así como el estado financiaba la atención en salud a casi toda la población, garantizaba la oferta de prestación de servicios y mantenía abiertas las instituciones de salud para quienes quisieran hacer uso de ellas mediante la solicitud respectiva. Además, el Estado pagaba el $100 \%$ de todos los gastos de funcionamiento institucional, remuneraba a todos sus trabajadores, trabajaran o no, toda vez que debían estar disponibles y atentos para servir a cada persona que demandara la atención en salud por motivos de enfermedad; de este modo garantizaba la oferta de servicios, y financiaba el 100\% del subsidio a la oferta.

En el año 1993 con la aprobación de la ley 100, la historia del sistema de salud en Colombia se dividió en dos etapas, antes y después de 1993; en este año el Sistema 
de Salud dio un giro de 360 grados, el estado cambio radicalmente y de manera progresiva la forma de prestación de los servicios de salud, en este nuevo esquema incluyó la obligatoriedad de realizar acciones de promoción de la salud, prevención de la enfermedad y su continuidad con los procesos de tratamiento y rehabilitación; de igual manera, cambió la forma de financiar las instituciones públicas, pues pasó de financiar la oferta a financiar la demanda (el subsidio a la oferta son los servicios que ofrecen las instituciones ya sea (IPS ó ESE públicas) y el Subsidio a la demanda son las necesidades que demanda el Usuario); es decir, comenzó a girar recursos de acuerdo al número de personas que no tenían ningún tipo de seguridad social, por decirlo de cierta forma, no pertenecían al régimen subsidiado, al cual debían afiliarse todas las personas que residieran en cada municipio y tuviera niveles de pobreza uno o dos del SISBEN.

El SISBEN es una encuesta que determina la situación socioeconómica de cada persona; es decir, mide el nivel de pobreza, y en consecuencia las personas más pobres deben afiliarse a las Administradoras de Régimen Subsidiado (ARS), empresas que reciben los recursos del estado, ya fuera a través del nivel nacional, departamental o municipal, (cofinanciado) para garantizar la atención en salud a este tipo de usuarios.

No obstante, los cambios de los subsidios a la demanda en detrimento de los subsidios a la oferta han mostrado hechos significativos; a la fecha en materia de cobertura, esta ha mostrado avances muy importantes; sin embargo, ha surgido un interrogante al interior de las autoridades vinculadas al ramo de la salud ¿Por qué a mayor cobertura, mayor enfermedad y mortalidad?.

Un hecho relevante respecto de la Ley 100 de 1993, fue que la salud pasó a convertirse en un derecho humano fundamental, convirtiéndose la consecución del nivel de salud más alto posible, en un objetivo social prioritario en todo el mundo, y para cuya realización se ha requerido de la acción de otros sectores sociales y económicos; además, con Ley 100/93, se definieron dos formas de ingresar al Sistema de Seguridad social en Salud, una de ellas es el Régimen Contributivo, al cual ingresan todas las personas que trabajan para una entidad pública o privada o que reciben un sueldo de un salario mínimo legal al mes y el Régimen Subsidiado, en el cuál ingresan todas las personas que por sus condiciones de pobreza y/o vulnerabilidad no pueden pagar una afiliación independiente.

En estos cambios, es importante reconocer los logros en materia de los derechos y deberes como ciudadanos y nuestra condición como sujetos de derechos en relación con el Estado. En tal sentido, tener salud no es solamente el resultado de factores biológico-genéticos, del medio ambiente y del comportamiento, sino que además involucra el deber del Estado de procurar los medios y los recursos necesarios para que la población pueda acceder a ella; en este orden de ideas, el estado Colombiano debe garantizar a todas las personas la seguridad social; así mismo, el derecho 
que asiste a los ciudadanos para acceder a los servicios de promoción, prevención, protección y recuperación de la salud, la cual debe ser garantizada por el mismo Estado; y así, quedó definida en la Constitución Política de 1991, en los artículos 48 y 49.

Es importante tener en cuenta que estas acciones orientadas al conjunto de la población, se deben complementar con aquellas orientadas al individuo, incluidas en el Plan Obligatorio de Salud POS, tanto en el régimen contributivo como en el subsidiado y que son de responsabilidad de las EPS y ARS. Estas son acciones que también buscan prevenir las enfermedades y promover la salud. La idea es que los ciudadanos tengan acceso al servicio, independientemente de su capacidad de pago o de su ubicación geográfica.

Tabla 4. Total Cobertura Régimen Subsidiado

\begin{tabular}{lcccccc}
\hline Municipios/periodo & $\mathbf{2 0 0 0}$ & $\mathbf{2 0 0 1}$ & $\mathbf{2 0 0 2}$ & $\mathbf{2 0 0 3}$ & $\mathbf{2 0 0 4}$ & $\mathbf{2 0 0 5}$ \\
\hline Bucaramanga & 33,9 & 39,9 & 42,6 & 36,0 & 76,6 & 59,5 \\
Barrancabermeja & 58,3 & 60,9 & 62,0 & 56,0 & 56,6 & 60,6 \\
Floridablanca & 23,5 & 24,8 & 27,7 & 30,0 & 60,9 & 49,2 \\
Total Departamental & 42,6 & 45,0 & 47,0 & 44,0 & 60,6 & 59,2 \\
\hline
\end{tabular}

Fuente: Secretaría de Salud Departamental. Cálculos autor.

En resumen, asumir la gestión pública de la salud significa unir todos los esfuerzos desde las instituciones y el Estado, con el objetivo de lograr el bienestar y mejoramiento de la calidad de vida de todos los miembros de la sociedad. En fin, no sobra decirlo, pero la calidad en la prestación del servicio de salud en la actualidad ha declinado de manera ostensible en términos de la eficacia, en verdad prevalece la parte financiera sobre los aspectos éticos y humanos de la salud.

\section{Conclusiones}

El estado colombiano registró durante la mayor parte del siglo XX un proceso continuo de centralización en lo político, administrativo y económico; solo a finales de los años setenta se observan tendencias de cambio; a mediados de la década de los setenta, cerca del 13\% de los ingresos corrientes del gobierno central se transfirieron a los gobiernos locales; ya para el año 2001 este mismo indicador mostraba que tal proporción había alcanzado el 50\%. A comienzos de los ochenta se crea la Misión de Finanzas Intergubernamentales, cuyo informe se encarga de cuestionar el sistema de transferencia existente en ese momento, afirmando que no tenía en cuenta entre 
otras, las necesidades fiscales, ni la capacidad, ni el esfuerzo fiscal, para lo cual sugiere una nueva fórmula para el cálculo del situado fiscal, donde se incluyen elementos más adecuados.

En el año de 1983 el proceso de descentralización se inicia con el fortalecimiento de los municipios, para lo cual se examina la redefinición de las relaciones entre la administración local y el gobierno central nacional, generando mayor autonomía a los entes territoriales y trasladándoles funciones y recursos desde el nivel central. No obstante, es la constitución política de Colombia la encargada de crear las bases mediante la cual se concedieron las responsabilidades y recursos a los gobiernos territoriales, que para el caso de los municipios estableció la participación de estos en los ingresos corrientes de la nación; su avance definitivo se consolidó a través de la ley 60 de 1993 y más adelante se reforzó con el acto legislativo 01 de 2001 que logró fortalecer los entes territoriales al amparo de la ley 715 de 2001.

Las transferencias del gobierno central nacional han sido de tiempo atrás la principal fuente de financiación de los entes municipales y por consiguiente se han convertido en el mecanismo transmisor de la descentralización, con los cuales se ha podido cumplir los objetivos trazados en materia de gasto público y en particular en los sectores de educación y salud. Los cambios trascendentales en estos sectores, permitió que los municipios asumieran de manera progresiva el manejo directo de estos servicios, con lo cual se logró mejorar de forma sustancial su prestación, aumentando los niveles de cobertura y calidad.

Las transferencias giradas por la nación para educación a los municipios de la muestra, evidenció la importancia de los centros urbanos de Bucaramanga, Barrancabermeja y Floridablanca, lo cual les permitió para el período analizado, absorber en promedio cerca del $92 \%$ por este concepto; en el caso de la salud el promedio ascendió al 69\%; por el lado del gasto público, Bucaramanga, Barrancabermeja y Floridablanca invirtieron en promedio para el período 1997 2005 el 90\% en educación y el $73 \%$ en salud.

La evolución del gasto público en términos de educación y salud entre 1997 y 2005 mostró resultados favorables, obteniendo beneficios asociados a aumentos significativos en materia de cobertura. Fue así como en educación la cobertura aumentó para los tres niveles básicos en el período 1997-2005, para preescolar entre el $82 \%$ y el $88 \%$, básica primaria, cercana al $100 \%$ y para secundaria entre el $72 \%$ y el $86 \%$.

En salud, la cobertura del régimen subsidiado por municipios mostró un comportamiento favorable, como resultado de las medidas implementadas al interior del sector y que estuvieron asociadas a procesos de vinculación de la población que aun no disfrutaba de este servicio; los resultados fueron positivos, siendo Bucaramanga y Barrancabermeja los de mayor dinamismo, a diferencia del municipio de Floridablanca que registró el menor crecimiento durante el periodo. 
La deserción escolar es un fenómeno complejo que deriva de múltiples causas que explican la inasistencia a la escuela; para algunos municipios del departamento de Santander la deserción escolar ha venido disminuyendo mientras la cobertura ha ido aumentado, todo como resultado de las estrategias adoptadas y entre las cuales merece destacarse el beneficio a los costos educativos, los bibliobancos, el subsidio de transporte, la alimentación escolar y entre otros los kits escolares, la entrega de dotación y uniformes.

Por el lado de la salud, los cambios en los subsidios a la demanda en detrimento de los subsidios a la oferta han mostrado avances significativos en materia de cobertura; un hecho relevante respecto de la Ley 100 de 1993, fue que la salud pasó a convertirse en un derecho humano fundamental, convirtiéndose la consecución del nivel de salud más alto posible, en un objetivo social prioritario en todo el mundo.

La focalización del gasto público es en apariencia la herramienta más eficaz en cuanto hace referencia al manejo y aplicación de los recursos estatales, ya que su efecto es muy positivo en el corto plazo, toda vez que destina los recursos a un grupo específico de la población (los pobres); no obstante, se deben mejorar las herramientas de análisis que permitan establecer con claridad esa franja de población más necesitada, con el fin de evitar caer en el error de no identificar los beneficiarios potenciales de un servicio en particular y que estos recursos lleguen a la población que en realidad no los necesita. En referencia a la alternativa de universalización en la aplicación del gasto, conviene señalar que esta puede llegar a ser en un momento determinado la más deseable, toda vez que con ella se invocaría el principio de igualdad de derechos; sin embargo, la limitada disponibilidad de recursos por parte del estado, hace que esta alternativa deba combinarse con adecuadas estrategias de focalización, de tal manera, que a medida que se focalice el gasto, poco a poco se logre obtener la cobertura total de la población; es más, podría pensarse en que estas dos alternativas al final no son excluyentes.

Los esfuerzos realizados por el Gobierno Central Nacional durante tres décadas de descentralización fiscal en materia de educación y salud, fueron de gran importancia para las regiones; de esta forma, el diseño de las políticas coordinadas por el gobierno nacional y los entes territoriales se orientaron específicamente a fortalecer los mecanismos más eficaces, permitiendo mejorar la prestación de estos servicios en cobertura y calidad. 


\section{Referencias}

Barón, Juan; Meisel, Adolfo, "La descentralización y las disparidades económicas regionales en Colombia en la década de 1990", en Documentos de trabajo sobre economía regional, núm. 36, Banco de la República, Cartagena, julio de 2003

Bonet, Jaime, "Inequidad espacial en la dotación educativa regional en Colombia”, en Documentos de trabajo sobre economía regional, núm. 56, Banco de la República, Cartagena, febrero de 2005

Bonet, Jaime, "Desequilibrios regionales en la política de descentralización en Colombia”, en Documentos de trabajo sobre economía regional, núm. 77, Banco de la República, Cartagena, octubre de 2006

Giraldo, Cesar, "Finanzas Públicas en América Latina: la economía política", Ediciones desde abajo, Bogotá, enero de 2005

Mineducación, "Balance del plan decenal de educación 1996-2005: La educación un compromiso de todos", Min educación, Bogotá, agosto de 2006

Restrepo, Darío; CUELLAR, Daniel, Historias de Descentralización. Transformación del Régimen Político y Cambio en el Modelo de Desarrollo, América Latina, Europa y EUA. "Economía política de las estructuras espaciales del estado Colombiano", junio de 2006. Centro de Investigaciones para el desarrollo CID. Universidad Nacional de Colombia. Bogotá, D.C.

Viloria, Joaquín, “Educación primaria en Cartagena: Análisis de cobertura, costos y eficiencia", en documentos de trabajo sobre economía regional, núm. 33, Banco de la República, Cartagena, octubre de 2002 